\title{
High-Throughput cDNA Screening Utilizing a Low Order Neural Network Filter
}

BioTechniques 21:1110-1114 (December 1996)

\author{
Guyang Matthew Huang, James Farkas and \\ Leroy Hood \\ University of Washington, Seattle, WA, USA
}

model of input/output relationships whose fidelity is dependent on network architecture. Complexity can gracefully be traded for accuracy, a desirable property for the screening application.

Our goal initially was to autonomously scan cDNA sequences for a single-spanning transmembrane region specifically for membrane proteins from target families. We focused on maximizing performance vs. cost and considered detailed sequence analysis a separate task. The performance goals for our filter were simply to identify the maximum number of single-spanning transmembrane like sequence segments while achieving a manageable false-alarm rate. For our effort, we endeavored to incorporate as much apriori biological, physical and annotated database information as possible in the neural network design and then utilize heuristic rules to examine the likelihood on the output.

Techniques for autonomous prediction of membrane-spanning domains in protein sequences have been discussed previously $(8,29)$. The transmembrane domain is characterized by the need for externally hydrophobic structures to span the lipid bilayer of the cell membrane. One or more alpha $(\alpha)$ helices are a typical motif, but beta $(\beta)$ sheets and amphipathic helices are also seen (14). Typically, a moving window of hydrophobicity value is computed, and a threshold value is selected to discriminate transmembrane domains. These filters are suitable for use as high-throughput screening filters but can show sensitivity to hydrophobic regions in general and can lead to a large number of false alarms, especially when scanning all potential reading frames for cDNA sequences. Preliminary screening of 500 cDNA sequences from our experimental database using a nine-peptide lumped hydrophobicity index resulted in several hundred reported detections. Recent results $(2,5,6,19,22,24,26)$ using a variety of techniques yield over $90 \%$ accuracy in transmembrane $\alpha$ helix prediction. These techniques attempt to model generalized structures and often utilize a computation-intensive multiple algorithm polling scheme that is implemented on a network server. Although extremely useful for detailed analysis, these techniques are not optimized for high-throughput screening.

\section{MATERIALS AND METHODS}

\section{Protein Model}

Use of amino acid (aa) positions in a learning algorithm requires that sufficient information is available in the training set and that the algorithm has sufficient freedom to infer and 
Table 1. Heuristic Rules for Augmenting Neural Network Filter

\begin{tabular}{|lc|}
\hline Rule & \multicolumn{1}{c|}{ Logic } \\
\hline Charged Residues & $\begin{array}{c}\text { If \# Arginine, Lysine, Aspartic } \\
\text { or Glutamic }>8 \text { reject }\end{array}$ \\
$\begin{array}{l}\text { Proline Content } \\
\text { Consecutive Prolines }\end{array}$ & $\begin{array}{c}\text { If } 5 \text { consecutive prolines then } \\
\text { reject }\end{array}$ \\
\hline
\end{tabular}

model any underlying physical processes from positional data alone. Limitations of such techniques for anonymous cDNA sequences have been noted (3). Use of physical properties associated with each residue to more directly model salient information has been utilized previously $(5,27)$ in statistical model formation. To reduce the complexity and increase the speed of our filter, we desired a one-dimensional protein sequence model that contained as much physical and positional information as possible. For our efforts in detecting transmembrane domains, we chose to represent the amino acids with their normalized hydrophobicity values from the Engelman scale (7). Future work in the area of simplified folding of potential function representations for protein sequences may enable more powerful low-dimensional pattern recogition fil- ters $(20,28)$. We then used a set of heuristic sanity rules to compensate for the inadequacies of our protein representation and for gaps in the training data. These content rules, shown in Table 1 , focus mainly on percentage of proline, the steric properties of which are unavailable to a filter seeing a hydrophobicity sequence.

\section{Training and Evaluation Set Design}

Design of our training set was driven by both the amount and quality of target data available. Experimental membrane protein structural data are sparse. A relatively large number of predicted single-spanning transmembrane domains are available in public databases. Annotated transmembrane regions for proteins whose sequence and function are well characterized and which are homologous across species were designated as our target feature. For our design, 359 proteins containing a single transmembrane region were identified from the $\mathrm{T}$ cell receptors (TCR), class I and II major histocompatibility antigens (MHC), immunoglobulin (IG) superfamily, lymphocyte CD antigens and other receptors containing annotated single-spanning membrane regions identified from SWISSPROT, Protein Information Resource (PIR $\left.{ }^{\circledR}\right)$, PROSITE and other databases. Of these, 80 proteins representing this 
sample space were selected and designated as a training set. Forty-three of the eighty were homologous to other training set members, with MHC I proteins represented by $28 \mathrm{mem}-$ bers and MHC II proteins by 14 . Two sets of 100 and 179 proteins, respectively, which had distinct identity but high homology to the training set, were designated as a first-stage design evaluation test set and a second-stage test set to evaluate the final design. For training, a window of 50 aa across the transmembrane domain was extracted from each training protein. Sixty counter examples of nonhomologous nontransmembrane $\alpha$ helices and regions of high hydrophobicity were included in the training set. A set of 22 SWISS-PROT proteins selected from the evaluation test used by Jones (17) and five porins with $\beta$ sheet transmembrane regions were designated a verification test set for detection performance. These proteins incorporate both single- and multiple-membrane spanning regions and were not homologous to proteins in either the training or the preliminary evaluation sets.

\section{Neural Network Design}

The design of network architecture was influenced both by the biology of the feature we were trying to detect and the requirements for accuracy and speed. The number of hidden layers in a neural network can be shown to correspond to the number of decision nodes required for a given classification problem (12). Rules characterizing single-spanning transmembrane regions have been identified (18) and serve as a basis for a transmembrane region identification heuristic decision tree. These rules are based on primary sequence relative positional relationships rather than derived secondary or tertiary structure correlations. The single level of primary sequence correlations indicated to us that only a single hidden layer in our neural network was required. Reliance on contextual data required spanning the region of interest and visibility on either side. We used a window of 50 aa to provide for a transmembrane region up to 35 -aa-long and at least 15 precursor extracellular amino acids. The size of this input window ensures that enough contextual data are presented to the modeling technique and in no way imposes constraints on the size of the transmembrane region model itself. A three-layer feed-forward neural network architecture was selected based on ease of training and performance/complexity tradeoff potential. Initially, twenty-five nodes in the hidden layer were used in the design with the intention of later minimizing the hidden layer nodes for use in high-throughput processing.

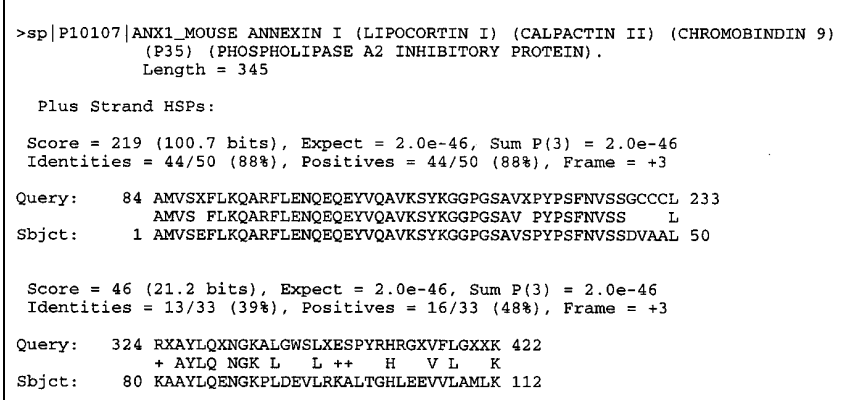

Figure 1. Annexin alignment.
Table 2. Observed and Predicted Transmembrane Regions from 50 Proteins

\begin{tabular}{|c|c|c|}
\hline Protein & Annotated TM start & Predicted TM start \\
\hline 4f2_human & 82 & 83 \\
\hline cek̄2_chick & 365 & 368 \\
\hline glp_pig & 63 & 62 \\
\hline glpa_human & 92 & 89 \\
\hline gmcr_human & 321 & 324 \\
\hline gplb_human & 148 & 146 \\
\hline hema_cdvo & 35 & 35 \\
\hline il2a_human & 241 & 237 \\
\hline lech_human & 40 & 31 \\
\hline leci_mouse & 40 & 54 \\
\hline magl_mouse & 517 & 510 \\
\hline motb_ecoli & 28 & 24 \\
\hline myp0_human & 154 & 155 \\
\hline nep_human & 28 & 24 \\
\hline trbm_human & 516 & 513 \\
\hline trsr_human & 63 & 62 \\
\hline vmt2_iaan & 25 & 27 \\
\hline \multirow[t]{4}{*}{ 5ht3_mouse } & 246 & 248 \\
\hline & 278 & \\
\hline & 306 & 355 \\
\hline & 465 & 457 \\
\hline \multirow[t]{3}{*}{ cb21_pea } & 62 & \\
\hline & 114 & \\
\hline & 182 & 183 \\
\hline \multirow[t]{7}{*}{ opsl_calvi } & 48 & \\
\hline & 85 & \\
\hline & 125 & \\
\hline & 164 & \\
\hline & 212 & 213 \\
\hline & 275 & 272 \\
\hline & 306 & \\
\hline \multirow[t]{6}{*}{ opsd_bovin } & 37 & 37 \\
\hline & 74 & 86 \\
\hline & 153 & 153 \\
\hline & 203 & 205 \\
\hline & 252 & 252 \\
\hline & 285 & \\
\hline
\end{tabular}

Sigmoid transfer functions and back-propagation learning (25) with momentum and bias were used for training. The single output of the network is a positive real number similarity value, which we compared to an empirically set threshold. Instead of including extra states in the neural network for reliability estimates as in other work (24), we used an empirically set threshold to discriminate suspected transmembrane-like regions and then only reported if the threshold was exceeded three times within a five-consecutive-aa region of the sequence being scanned.

The initial network was trained, and the resulting weights were incorporated into a standalone screening program, which scanned a moving input window over a protein sequence incrementing a residue at a time. For the initial training set, matches with extracellular $\alpha$ helices and signal 
Table 3. SWISS-PROT Porin Predicted Transmembrane Location and Observed Computation Time

\begin{tabular}{|lccc|}
\hline & $\begin{array}{c}\text { Neural } \\
\text { Net }\end{array}$ & Phd & TMpredict \\
\hline nmpc_ecoli & 193 & ----- & ----- \\
pori_bppa2 & 193 & ----- & ----- \\
porf_pseae & 177 & ----- & ----- \\
& 223 & ----- & ----- \\
& 269 & ----- & ----- \\
om32_comac & ----- & ----- & ---- \\
porf_psesy & ---- & ----- & --- \\
seconds/peptide & .013 & 1.590 & .018 \\
\hline
\end{tabular}

sequences resulted in a probability of false alarm of 0.23 for a threshold value that detected all the transmembrane region. The training set was augmented with an additional 13 counter examples of non-transmembrane $\alpha$ helices taken from ribosomal proteins. The trained filter was then applied to a firststage test set of 100 proteins with a resulting probability of false alarm of 0.04 for a probability of detection of 0.99 .

The free parameter in our design was the number of nodes in the hidden layer. Due to the requirement to minimize the number of multiplications, the effect of eliminating hidden layer nodes was evaluated. The network essentially became untrainable with less than three hidden nodes. Reduced networks with as little as four hidden nodes resulted in quasi linear increases in root means square (rms) error during training. We selected six hidden nodes as a good compromise between speed and accuracy. Performance of the minimized net against the second-stage set of check protein sequences resulted in a probability of detection of 0.98 with a probability of false alarm (positive for nontransmembrane regions) of 0.06. Examination of the network weights showed no extrenal values or obvious rules.

Table 2 shows results for the final evaluation single-spanning proteins and a few representative multiple-spanning proteins. Our filter, which was designed to try to exclude multiple-spanning regions, identified all but one of the singlespanning domains and all of the multiple-spanning transmembrane regions where the domains were separated by greater than 50 aa. We also identified the transmembrane regions in immunoglobulin G-binding protein precursor (iggb_strsp) and melittin, which gave some difficulty for the helix-specific algorithm of Rost et al. (24). The false-alarm rate for the verification test set was $6 \%$. The filter also identified three of the five porin $\beta$ sheet membrane proteins presented to it as shown in Table 3. A false-alarm check set of 100 globular, extracellular alpha and cytoplasmic protein segments were chosen from the SWISS-PROT database and resulted in a false-positive probability of 0.08 .

\section{RESULTS AND DISCUSSION}

The filter was then applied to an existing database of 2123 anonymous cDNA sequences. A total of 61 sequence/reading frame transmembrane detections were identified. Detections from the cDNA library were subjected to a blastx and blastn
(1) similarity search utilizing the National Center for Biotechnology Information (NCBI) network server to determine similarity to a known transmembrane protein in the predicted reading frame. The 61 detections produced 15 cases of highscoring homology segments in the correct reading frame. Twelve of these cases were associated with membrane-spanning proteins, five of these having over $50 \%$ similarity in a known membrane-spanning region, while the remainder had no homologs. An alignment of a filter transmembrane detection report at aa 32 with annexin I, a protein that binds reversibly with phopholipid bilayers (PROSITE pattern database No. PS00223), is seen in Figure 1. The homologous region is in one of the conserved repeats in the annexin family, thought to be a phospholipid binding site. The detected transmembrane-like regions were input to the high order neural network-based Phd algorithm (24) and TMpredict (10) algorithm, which is based on a database of natural-occurring transmembrane regions. Of the 61 detections identified by our filter, the Phd algorithm identified 13 of these as strongly transmembrane, 28 as medium-to-low confidence predictions, while 20 generated no similarity. Thirty-nine of our detections were also designated transmembrane by TMpredict. A benchmark set of protein queries was used to evaluate the effective throughput of our filter and the network server 
versions of Phd and TMpredict. Table 3 summarizes the results of this comparison for porins. Here, we compared the performance of three algorithms on human transferrin receptor and porin proteins. The transferrin receptor has a transmembrane region experimentally identified at aa 62-88 (16). All three algorithms predict the transmembrane domain reasonably well. Although strong experimental data of $\beta$ sheet type transmembrane regions is lacking, these proteins have been localized to the outer membrane and show low propensity for helix formation. Porin is a bacterial and mitochondrial outer membrane channel protein with $\beta$-strand structure $(4,21)$. Our filter identified three of the five porin proteins presented to it, which were not detected by the other two methods $(23,24)$. Table 3 shows the average computation time per peptide. This data reflects performance utilizing the Perl language implementation of our network, which is available through the file transfer protocol (ftp). Execution speed can be increased by an order of magnitude if the filter is implemented in a compiled language such as $\mathrm{C}$. Computation times are estimated from the time notations on the returned results. Network service delays on the order of hours are encountered in many e-mail-based analysis servers.

The main result of this work is that it provides some indication of the performance of modern pattern-recognition techniques for use in rapidly scanning anonymous sequences. Even though trained on a relatively small set of annotated database examples, a low order neural network-based filter can produce useful results in rapidly identifying sequence features.

\section{REFERENCES}

1.Altschul, S.F., W. Gish, W. Miller, E.W. Myers and D.J. Lipman. 1990. Basic local alignment search tool. J. Mol. Biol. 215:403-410.

2.Chou, P.Y. and G.D. Fasman. 1974. Conformational parameters for amino acids in helical, beta sheet, and random coil region calculated from proteins. Biochemistry 13:211-221.

3.Cohen, B., S. Presnell and F. Cohen. 1993. Origins of structural diversity within sequentially identical hexapeptides. Protein Sci. 2:2134-2145.

4.De Pinto, V.D. and F. Palmieri. 1992. Transmembrane arrangement of mitochondrial porin or voltage-dependent anion channel (VDAC). J. Bioenerg. Biomembr. 24:21-26.

5.Dombi, G.W. and J. Lawrence. 1994. Analysis of protein transmembrane helical regions by a neural network. Protein Sci. 3:557-566.

6.Eisenberg, D., E. Schwartz, M. Komaromy and R. Wall. 1984. Analysis of membrane and surface protein sequences with the hydrophobic moment plot. J. Mol. Biol. 179:125-142.

7.Engelman, D.M., T.A. Steitz and A. Goldman. 1986. Identifying nonpolar transbilayer helices in amino acid sequences of membrane proteins. Annu. Rev. Biophys. Biophys. Chem. 15:321-353.

8.Fasman, G.D. and W.A. Gilbert. 1990. The prediction of transmembrane protein sequences and their conformation: an evaluation. Trends Biochem. Sci. 15:89-92.

9.Ferran, E.A. and B. Pflugfelder. 1993. A hybrid method to cluster protein sequences based on statistics and artificial neural networks. Comput. Appl. Biosci. 9:671-680.

10.Hofmann, K. and W. Stoffel. 1993. TMbase - A database of membrane spanning proteins segments. Biol. Chem. Hoppe Seyler 347:166.

11.Holley, L.H. and M. Karplus. 1991. Neural networks for protein structure prediction. Methods Enzymol. 202:204-224.

12.Hopfield, J.J. 1982. Neural networks and physical systems with emergent collactive computational abilities. Proc. Natl. Acad. Sci. USA 79:25542558.

13.Huang, G.M., D. Gordon, J. Farkas and L. Hood. 1994. Above and beyond database - A high throughput system for cDNA sequence analysis. Genome Science and Technology Conference VI, Hilton Head Island, SC. p. 62 .

14.Hucho, F., U. Gorne-Tschelnokow and A. Strecker. 1994. Beta structure in the membrane spanning part of the nicotinic acetylcholine receptor (or how helical are transmembrane helices?). Trends Biochem. Sci. 19:383-387.

15.Hunkapiller, T., R. Kaiser, B. Koop and L. Hood. 1991. Large scale and automated DNA sequence determination. Science 254:59-67.

16.Jing, S.Q. and I.S. Trowbridge. 1987. Identification of the intermolecular disulfide bonds of the human transferrin receptor and its lipid-attachment site. EMBO J. 6:327-331

17.Jones, D.T., W.R. Taylor and J.M. Thornten. 1994. A model recognition approach to the prediction of all-helical membrane protein structure and topology. Biochemistry 33:3038-3049.

18.Landolt-Marticorena, C., K.A. Williams, C.M. Deber and A.F. Reithmeir. 1993. Nonrandom distribution of amino acids in the transmembrane segments of human type I single span membrane proteins. J. Mol. Biol. 229:602-608

19.Lohmann, R., G. Schneider, D. Behrens and P. Wrede. 1994. A neural network model for the prediction of membrane spanning amino acid sequences. Protein Sci. 3:1597-1601.

20.Maiorov, V. and G. Crippen. 1994. Learning about protein folding via potential functions. Proteins 20:167-173.

21.Nikaido, H. 1993. Transport across the bacterial outer membrane. J. Bioenerg. Biomembr. 25:581-589.

22.Person, B. and P. Argos. 1994. Prediction of transmembrane segments in proteins utilizing multiple sequence alignments. J. Mol. Biol. 237:182192.

23.Qian, N. and T.J. Sejnowski. 1988. Predicting the secondary structure of globular proteins using neural network models. J. Mol. Biol. 202:865-884.

24.Rost, B., R. Casadio, P. Fariselli and C. Sander. 1995. Transmembrane helices predicted at 95\% accuracy. Protein Sci. 48:521-533.

25.Rumbelhart, D.E. 1986. Parallel Distribution Processing: Explorations in the Microstructure of Cognition. MIT Press. Cambridge.

26.Sipos, L. and G. von Heije. 1993. Predicting the topology of eukaryotic membrane proteins. Eur. J. Biochem. 213:1333-1340.

27.Vanhala, J. and K. Kaski. 1993. Protein structure prediction system based on artificial neural networks. Proc. First Intl. Conf. Intelligent Sys. Mol. Biology. AAAI 1993:402-410.

28.Wallqvist, A. and M. Ullner. 1994. A simplified amino acid potential for use in structure predictions in proteins. Proteins 18:267-280.

29.Weiss, S., D. Cohen and N. Indurkhya. 1993. Transmembrane segment prediction from protein sequence data. Proc. First Intl. Conf. Intelligent Sys. Mol. Biology. AAAI 1993:420-428.

30.Wu, C., M. Berry, Y. Fung and J. McLarty. 1993. Neural networks for molecular sequence classification. Proc. First Intl. Conf. Intelligent Sys. Mol. Biology. AAAI 1993:429-436.

Received 19 August 1996; accepted 24 October 1996.

Address correspondence to:

G. Matthew Huang

DuPont Agriculture Biotechnology

1 Innovation Way, Suite 100

Delaware Technology Park

Newark, DE 19711, USA

Internet: huanggm@cdna.dt.dupont.com 